\title{
Microbiological quality of grain cultivated in the North Caucasus region
}

\author{
Gagkaeva T.Yu. ${ }^{1}$, Gavrilova O.P. ${ }^{l}$, Orina A.S. ${ }^{1}$, Burkin A.A. ${ }^{2}$, Khusaynov Kh.A. ${ }^{3}$
}

${ }^{1}$ All-Russian Institute of Plant Protection (VIZR), St. Petersburg, Russia; ${ }^{2}$ All-Russian Research Institute for Veterinary Sanitation, Hygiene and Ecology, Moscow, Russia; ${ }^{3}$ Chechen Research Institute of Agriculture, Grozny,

Gikalo village, Russia

E-mail: t.gagkaeva@mail.ru

Key message. The wheat and barley grain harvested in North Caucasus in 2019 were mostly infected by Alternaria (to 93\%) and Fusarium (to 14\%) fungi. The risk of contamination of grain with mycotoxins was shown.

Keywords: fungi, Alternaria, Fusarium, DNA, mycotoxins

Widespread groups of Alternaria and Fusarium fungi contaminate grain and other crops. Alternaria fungi are usually not causing apparent harm to host, but some Alternaria species can produce mycotoxins, among which alternariol (AOH) is widely present in agricultural products. The most important mycotoxins in cereals are deoxynivalenol (DON), T-2 toxin and zearalenone (ZEA) produced by Fusarium fungi. The mycotoxin production of fungi depends on climatic and geographical factors. The influence of natural environmental conditions in some areas of North Caucasus region on the fungal infection and contamination with mycotoxins of cereals is poorly studied.

The aim of study was to evaluate the microbiological quality and to examine amounts of the mycotoxins in grain grown in the North Caucasus region.

Total twenty wheat and three barley grain samples harvested in 2019 were analyzed. They were originated from Chechen Republic ( $n=19)$, Republic of Ingushetia $(n=3)$ and Republic of Dagestan $(n=1)$. This territory is characterized by diverse ecosystems and, according to this, 8 samples in the dry steppe, 5 in the steppe, 8 in the forest steppe and two in the mountain forest were cultivated.

The percentage of infected grains was evaluated by mycological method. The DNA content of the group of Fusarium species producing trichothecene mycotoxins (Tri-Fusarium) and the DNA content of the Alternaria section Alternaria fungi was determined using quantitative PCR. The amount of mycotoxins in the grain were analysed by the certified test-systems for the ELISA according to the protocol of manufacturer (VNIIVSGE, Russia).

Alternaria and Fusarium fungi were the most abundant genera in the mycobiota associated with grain. The infection grain by Alternaria species varied in the range of 55-93\%. The proportion of the species section Alternaria (94\%) prevailed over section Infectoriae (6\%). The maximum Fusarium infection of the grain reached to $14 \%$ in wheat from the dry steppe zone. Arid conditions of the growing season 2019 resulted in lower infection with $F$. graminearum, which is typical pathogen in the North Caucasus region. This species was identified in 30\% of samples with a range of infection of 1-6\%. The main importance of observation of the study was identification of $F$. langsethiae, which is strong producer of T-2 toxin, in $13 \%$ of grain samples grown in the Chechen Republic, where this species was not previously described. The other producer of this mycotoxin $-F$. sporotrichioides was revealed in $22 \%$ samples.

DNA of Alternaria fungi was detected in all grain samples in the range of $4.3 \times 10^{-3}-7.5 \times 10^{-2} \%$ of the total DNA without any differences in the amounts between the ecosystems. DNA of Tri-Fusarium fungi were detected in 7 samples, the amounts of fungal biomass ranged from $3.1 \times 10^{-3}$ to $2.9 \times 10^{-2} \%$ of the total DNA.

Mycotoxin AOH was detected in $65 \%$ of samples in the amounts of $11-675 \mathrm{ppb}$. Among the analysed samples only the grain originated from the steppe and dry steppe zones was free from AOH. DON was detected in one wheat grain sample from the forest-steppe in the amount of $40 \mathrm{ppb}$. ZEN was found in three wheat samples from the steppe and forest-steppe zones, in amounts 10-40 ppb. The occurrence of T-2 toxin was revealed in $26 \%$ of the samples. The contamination by this mycotoxin varied from 7 to $650 \mathrm{ppb}$. The maximum of T-2 toxin was detected in barley grain from the dry steppe zone, and it exceeded the permitted level for this mycotoxin in Russia in 6.5 times.

Positive correlations of the percentage of infected grains with Alternaria fungi with the amount of DNA of Alternaria section Alternaria $(\mathrm{r}=0.47, \mathrm{p}<0.05)$ as well as with content of $\mathrm{AOH}(\mathrm{r}=0.46, \mathrm{p}<0.05)$ were established. Also, the positive correlation between the amount of Tri-Fusarium DNA and both DON and ZEN amounts ( $\mathrm{r}=0.88$ and $\mathrm{r}=0.79, \mathrm{p}<0.05)$ was revealed. Moreover, the positive correlation between the amounts of these two mycotoxins and the infection of grain with $F$. graminearum that producer of DON and ZEN $(r=0.64$ and $\mathrm{r}=0.56, \mathrm{p}<0.05)$ was found. A relationship between occurrence of Fusarium species producing T-2 toxin and amounts of this mycotoxin in grain was not detected.

This study has shown that even in a dry growing season there is a risk that grain may become contaminated with mycotoxins, in particular toxins of Alternaria and Fusarium species, which do not require high humidity. At the same time, the presence of pathogenic $F$. graminearum in the grain suggests, that in growing seasons with sufficient moisture, a contamination of grain by DON and ZEN can be significantly above the maximum permitted levels of these mycotoxins.

The study was supported by the Russian Science Foundation (project no. 19-76-30005). 